1 Analysis of Muscle Synergies and Activation-Deactivation Patterns in

2 Subjects with Anterior Cruciate Ligament Deficiency during Walking

3 Gil Serrancolí ${ }^{1}$, Joan C. Monllau ${ }^{2,3}$, Josep M. Font-Llagunes ${ }^{1}$

$4{ }^{1}$ Department of Mechanical Engineering and Biomedical Engineering Research Centre,

5 Universitat Politècnica de Catalunya, Diagonal 647, 08028 Barcelona, Catalonia.

$6{ }^{2}$ Department of Orthopaedic Surgery, Hospital del Mar, Universitat Autònoma de Barcelona,

7 Passeig Marítim 25-29, 08003 Barcelona, Catalonia.

$8{ }^{3}$ Institut Català de Traumatologia i Medicina de l'Esport. Hospital Universitari Quirón9 Dexeus, Sabino de Arana 5-19, 08028 Barcelona, Catalonia.

Corresponding author: Gil Serrancolí.

Department of Mechanical Engineering and Biomedical Engineering Research Centre,

Number of words in the abstract: 194

Number of words in the main text: 4445 


\section{Analysis of Muscle Synergies and Activation-Deactivation Patterns in \\ 2 Subjects with Anterior Cruciate Ligament Deficiency during Walking}

3

4

Background: The knowledge of muscle activation patterns when doing a certain task in subjects with anterior cruciate ligament deficiency could help to improve their rehabilitation treatment. The goal of this study is to identify differences in such patterns between anterior cruciate ligament-deficient and healthy subjects during walking. Methods: Electromyographic data for eight muscles were measured in a sample of eighteen subjects with anterior cruciate ligament deficiency, in both injured (Ipsilateral group) and non-injured (Contralateral group) legs, and a sample of ten healthy subjects (Control group). The analysis was carried out at two levels: activation-deactivation patterns and muscle synergies. Muscle synergy components were calculated using a non-negative matrix factorization algorithm.

Findings: The results showed that there was a higher co-contraction in injured than in healthy subjects. Although all muscles are activated similarly since all subjects developed the same task (walking), some differences could be observed among analyzed groups.

Interpretation: The observed differences in the synergy components of injured subjects suggested that those individuals alter muscle activation patterns to stabilize the knee joint. This analysis could provide valuable information for the physiotherapist to identify alterations in muscle activation patterns during the follow-up of the subject's rehabilitation.

Keywords: ACL-deficiency, muscle synergies, muscle activation pattern, non-negative matrix factorization, electromyography 


\section{Introduction}

The rupture of the anterior cruciate ligament (ACL) is one of the most common knee injuries. It affects around two million people worldwide every year (Renström, 2012). Subjects with ACL deficiency alter their muscle activations when doing a certain task due to the lack of ACL. It is believed that muscles are activated synergistically following a certain pattern depending on the motor task (Lacquaniti et al., 2012; Ting and McKay, 2007; Ting, 2007; Ting et al., 2012), that is to say, our Central Nervous System (CNS) does not activate the muscles independently. Muscle synergies are represented by modules consisting of one Neural Command (NC), which represents the time activation of a set of muscles, and one Synergy Vector (SV), which represents the weighting factor of each muscle to its NC (Ting and Macpherson, 2005). The number of NCs is lower than the number of muscles. Therefore, the analysis of this lower dimensional activation pattern may explain the changes in neuromuscular activity due to the ACL rupture.

It is believed that the number of synergies used by a human being when walking is between 4 and 6 (Allen and Neptune, 2012; Clark et al., 2010; De Groote et al., 2014; Ivanenko et al., 2005, 2004; Oliveira et al., 2014). The Variance Accounted For (VAF) between the reconstructed and the original signals is evaluated to select the proper number of modules to be used when factorizing the signals. Most authors consider that a VAF $>0.9$ is the threshold to accept the reconstruction (Clark et al., 2010; Oliveira et al., 2014). It is reported that there are similarities in the muscle synergies when performing the same movement across subjects. Several authors reported muscle synergies when walking (Clark et al., 2010; Dominici et al., 2011; Ivanenko et al., 2004; Neptune et al., 2009; Oliveira et al., 2014), walking with perturbations (Ivanenko et al., 2005) or performing other tasks (Rugy et al., 2013). Clark et al. (2010) applied the muscle synergy analysis in post-stroke injured subjects. They observed that, although the patterns were similar among groups, the complexity in poststroke injured subjects was lower than in healthy subjects, i.e., they needed fewer modules to 
have a good signal reconstruction. It is unclear what synergistic strategy is followed by jointinjured subjects to activate the muscles spanning that joint. Depending on the joint injury, subjects can apply different activation strategies to avoid pain or to stabilize the joint.

Apart from the clinical evaluation of muscle co-contraction, the use of the factorization can be useful for motion analysis and simulation. There is indeterminacy when calculating the muscle forces, since they cannot be calculated experimentally due to invasiveness. The usual method to estimate the forces is with the resolution of an optimization problem (Erdemir et al., 2007), which consists of minimizing a cost function (a physiological variable) that represents the strategy of the CNS to activate the muscles. The optimization results can produce multiple physiologically feasible solutions due to the muscle redundancy. Some authors used the muscle synergy components to decrease the indeterminacy in the muscle force calculations, either in forward dynamics (Allen and Neptune, 2012; Neptune et al., 2009) or inverse dynamics (Walter et al., 2014) approaches. Regarding subjects with ACL deficiency, differences have been observed at the joint level as well as at individual EMG signals (Houck et al., 2007; Knoll et al., 2004; Rudolph et al., 2001; Serrancoli et al., 2014). As far as the authors know, the muscle synergy analysis has not been applied yet to subjects with this kind of injury. In consideration of that, this study could be useful at two levels. On the one hand, in a clinical application it would allow the specialist to follow the rehabilitation process of injured subjects. On the other hand, in a motion dynamic analysis, muscle synergies could be used to decrease the indeterminacy in the muscle force calculation of subjects with ACL deficiency.

The main goal of this study is to evaluate and compare the muscle activation patterns in healthy and injured subjects during walking. In particular, the analysis is carried out at two levels: activation-deactivation patterns and muscle synergies. In our study, all subjects with ACL deficiency were considered adapters (Button et al., 2006) and the measures were done a 
1 few days or weeks before the surgery of the ligament reconstruction. Although muscle synergy patterns can present many similarities among groups, since all of them perform the same task, human gait, our hypothesis was that the pattern of muscle synergy components may have different tendencies. As mentioned, there are studies that evaluate individual muscle activations in subjects with ACL deficiency, but the objective of this study is to evaluate the differences in muscle synergies compared to healthy subjects in order to better understand the muscle activation pattern in absence of ACL function. The knowledge of the differences in muscle synergies for subjects with ACL deficiency could help a physiotherapist to redirect the rehabilitation treatment. The analysis comprises two steps. The first is a comparison of the activation-deactivation pattern among healthy legs (Control group), injured subjects' injured legs (Ipsilateral group) and injured subjects' non-injured legs (Contralateral group). Then, a muscle synergy analysis is reported and compared among the three groups.

\section{Methods}

\subsection{Subjects}

Ten healthy subjects, five men and five women (mean (SD): age 31.5 (12.9) years, mass 65.2 (7.6) $\mathrm{kg}$, height $170.4(8.6) \mathrm{cm}$ ), and eighteen subjects with ACL deficiency, twelve men and six women (mean (SD): age 32.3 (10.99) years, mass $68.5(9.7) \mathrm{kg}$, height $172.1(7.6) \mathrm{cm}$ ), volunteered as participants in this study. No healthy subjects suffered any lower-limb injury. The injured subjects were classified as adapters, according to the medical staff and the widely used classification presented in (Button et al., 2006), which considers that they can be divided in three groups: copers, who return to the preinjury level of their daily tasks and sport activities; non-copers, who cannot return to their preinjury level of tasks and sport activities and have episodes of full giving way even in daily tasks; and adapters, who reduce or modify certain tasks or the sport level to prevent their knee giving way. All injured subjects reported 
1 that they could deal with daily life and they did not suffer pain when normal walking,

2 however, they felt discomfort and pain when they did sports that required knee pivoting, such as football or skiing. The time interval from the injury varied from one month to three years (mean (SD): 10.3 (12.0) months). All subjects provided their consent to contribute to this study.

\subsection{Experimental setup}

All volunteers were asked to walk a minimum of three overground gait cycles at a selfselected speed (mean (SD): $0.77(0.12) \mathrm{m} / \mathrm{s}$ healthy subjects and $0.80(0.13) \mathrm{m} / \mathrm{s}$ injured subjects). One of the gait cycles was selected from the recorded trials and was analyzed.

EMG data from sixteen muscles were measured with sixteen surface EMG sensors (Biometrics, Newport, United Kingdom) at $1000 \mathrm{~Hz}$. The signal of eight lower-limb muscles from each leg of the subjects was measured (Tibialis Anterior -TA-, Soleus -SO-, Gastrocnemius Lateralis -GL-, Gluteus Maximus -GM-, Rectus Femoris -RF-, Vastus Lateralis -VL-, Semitendinosus -ST- and Extensor Digitorus Longus -ED-). These muscles are the main contributors to the human walking. The EMG data for each subject (right and left leg) came from the same gait trial, which decreases the variability due to the differences that could appear when measuring different gait trials separately.

EMG signals were demeaned, rectified and filtered with a Butterworth low-pass filter at $6 \mathrm{~Hz}$. Then, they were normalized by Maximum Voluntary Contraction (MVC) values obtained by MVC exercises previously done. The exercises were selected to calculate the maximum muscle excitations (Kendall et al., 1993). The volunteers were asked to apply force against a resistance along a direction to activate the muscles responsible for: ankle plantar flexion/dorsiflexion (ED, SO, TA, GL), knee flexion/extension and abduction/adduction (RF, ST and VL) and hip flexion/extension and abduction/adduction (GM, ST and RF). Data from these trials were processed in the same way that walking trials (demeaned, rectified and 
1 filtered at $6 \mathrm{~Hz}$ ). The maximum values of EMG were selected among all available trials

2 (MVC exercises and gait trials). These values were verified visually and individually in each subject to avoid the acceptance of a wrong maximum value. All MVC exercises, as well as verifications, were carried out by the same technician to standardize the comparison. Using this normalization, the signal was constrained to be between 0 (not activated) and 1 (maximum activation). So, an activation close to 1 would mean that the muscle is near to its maximum activation.

Ground Reaction Forces (GRF) and marker trajectories were also measured to identify the events of the gait cycle. The GRF were measured by means of two force plates (AMTI, Watertown, MA) at $100 \mathrm{~Hz}$. Two marker trajectories from each foot (heel and tip of the first metatarsal bone) were captured by fourteen infrared cameras (Naturalpoint, Corvallis, OR). Once the gait cycle was identified for each leg, data was interpolated to 101 frames. Normalized EMG data are available on the net as supplementary data.

\subsection{Data Analysis}

Data analysis was carried out by means of MATLAB 7.10 v. R2010a (Mathworks, Natick, MA). All data were divided in three groups: Control, which consists of data from healthy subjects; Ipsilateral, from the ipsilateral leg, which is affected by the ACL injury; and Contralateral, from the non-injured leg of the subjects with ACL deficiency.

\subsubsection{Activation-deactivation pattern}

An initial analysis of the activation-deactivation pattern for each muscle was carried out to identify the differences in the activation timing between groups. The onset-offset activation pattern was calculated for each subject, considering EMG signal to be activated when it was higher than the following threshold:

$$
\text { Threshold }_{\text {on-off }}=\min (E M G)+0.5(\max (E M G)-\min (E M G))
$$


1 where EMG stands for an EMG signal. The activation pattern was calculated for each group.

2 A muscle was considered to be active when more than $50 \%$ of the subjects had this muscle

3 activated at a particular time frame.

4

5

6

7

\subsubsection{Non-negative matrix factorization}

EMG processed signals were factorized applying the Non-Negative Matrix Factorization (NNMF) to obtain the muscle synergy components: Neural Commands (NCs) and Synergy Vectors (SVs) (Lee and Seung, 1999; Ting and Macpherson, 2005). The factorization consists of decomposing the matrix containing all EMG signals (nframes $\mathrm{x}$ nmuscles) by two matrices: the NCs which account for the time activation of each module (nframes x nmodules) and SVs, which contain the weights of each muscle to each module (nmodules $\mathrm{x}$ nmuscles). This decomposition allows to obtain positive values for both NCs and SVs, what leads to a more physiological interpretation (Ting et al., 2012). The maximum value of each SV was constrained to be 1 to decrease the indeterminacy of the factorization. The NNMF was applied six times per trial, decomposing the signal in 1 to 6 modules.

\subsubsection{Comparison of results}

The match of the reconstructed EMG with the experimental one was evaluated by means of the Variance Accounted For (VAF) value, calculated as follows:

$$
V A F=1-\frac{\sum_{t=1}^{101} E M G_{t}^{r e c}-E M G_{t}^{e x p}}{\sum_{t=1}^{101} E M G_{t}^{e x p}}
$$

where $E M G_{t}^{r e c}$ and $E M G_{t}^{\exp }$ stand for the reconstructed and the experimental EMG signals at frame $t$ respectively. In order to compare statistically whether two values from two different samples were different (such as the comparison of VAF values), a t-test analysis was carried out and a p-value was calculated. If p-value $<0.05$, then the values were considered to be 
statistically different.

The differences in the tendency of SVs and NCs were measured by means of the Pearson's correlation coefficient (Clark et al., 2010). If this value was close to 1 , it meant that the shape of the compared sets of data was similar. In order to identify whether two NCs or SVs were statistically correlated, the threshold of the p-value was set equal to 0.001 .

\section{Results}

\subsection{Activation-deactivation pattern}

Figure 1 shows the activation-deactivation pattern of the eight analyzed muscles for the three data groups. It is remarkable that Ipsilateral and Contralateral TA showed a longer activation during the early stance phase $(0-40 \%)$. Moreover, the Ipsilateral's TA was active during all swing phase whereas Control's and Contralateral's TA activation just appeared in the beginning and at the end of this phase. SO and GL were only activated during the stance phase and both were activated earlier in Ipsilateral and Contralateral groups (10\% and 14\% of the gait cycle earlier respectively for SO, and $12 \%$ and $14 \%$ for GL). GM activation was slightly longer (6\% of the gait cycle) in the Ipsilateral leg. Regarding the knee muscles, it can be observed that the co-contraction of the injured subjects' Quadriceps (RF and VL) and Hamstrings (ST) was longer during the stance phase (5\% longer regarding the Contralateral and $9 \%$ regarding the Inspilateral group). Finally, Ipsilateral's ED activation pattern was slightly different from the other two groups, since both activation and deactivation of the injured subjects' ED appeared earlier than in the other two groups (at $14 \%$ and $62 \%$ of the gait cycle respectively).

\subsection{Analysis of dimensionality}

The similarity between the experimental and the reconstructed EMG signals was measured 
using the VAF value. The t-tests to compare the VAF values of the three groups showed that there were no significant differences in the dimensionality among groups, neither using 4 modules ( $p=0.20$ Control vs. Ipsilateral, $p=0.15$ Control vs. Contralateral and $p=0.82$ Ipsilateral vs. Contralateral) nor 5 modules ( $p=0.96$ Control vs. Ipsilateral, $p=0.82$ Control vs. Contralateral and $p=0.69$ Ipsilateral vs. Contralateral). Control group had a higher VAF in some muscles (such as GL, ST and ED when using 5 modules), as well as injured subjects in others (such as GM and VL when using 5 modules).

The reconstructed EMG signal reproduced the experimental one with a mean VAF value higher than 0.8 for all eight muscles using 4 modules (Figure 2). However, VL and ST signals were reconstructed with a mean $\mathrm{VAF}<0.9$. The use of a fifth module increases all VAF values, and in this case, the values of VAF were higher than 0.9 in all muscles. Therefore, in this study, 5 motor modules (synergies) were selected to compare the modules among groups (Figure 3).

\subsection{Variability intra-groups}

The number of modules was fixed to 5 and the cross correlation of the NCs and SVs between modules was analyzed within each group as in (Clark et al., 2010). Table 1 shows that the correlation among modules within each group was overall low, which means that the SVs and the NCs of the modules were independent from each other.

However, there were some slight similarities (significant positive correlations) in the shape of the NCs between modules 3 and 4, and modules 1 and 4 in the Control group $(r=0.58$ and $r=0.52$ respectively); between modules 1 and 3, and modules 1 and 4 in the Ipsilateral group ( $r=0.34$ and $r=0.56$ respectively); as well as between modules 1 and 3,1 and 4 , in the Contralateral group ( $r=0.37$ and $r=0.82$ respectively).

\subsection{Variability inter-groups}


1 Figures 4 to 6 show the comparison between NCs and SVs from two groups (Control vs.

2 Ipsilateral, Control vs. Contralateral and Ipsilateral vs. Contralateral, respectively). Although

3 the tendencies of SVs and NCs were similar in all groups, there were some differences that

4 were quantified in Table 2 through Pearson's correlation coefficients.

The comparison of all SVs from the same module between Control and Ipsilateral groups shows that they follow the same trend. However, in modules 1 and 4, the $r$ values were lower than 0.9 (Table 2). In module 4, the mean TA and RF components of the SV were significantly higher in the Ipsilateral group ( $p=0.03$ and $p=0.01$ respectively). It is also observed that during the stance phase in module 3 (basically TA activation), the NC was lower in the Control group ( $r=0.69)$. A similar result was obtained in module $1(r=0.77)$.

There were no significant differences in the pattern of SV between the Control and the Contralateral groups ( $r>0.9$ in all modules), but there were differences in the NCs. The shape of the third $\mathrm{NC}$ of the Contralateral group was quite different from the Control group $(r=0.43)$. Like in the Ipsilateral group, modules 1 and 3 of the Contralateral group were higher than those of the Control group at the early beginning of the stance phase.

Mean SV values between the Ipsilateral and Contralateral legs were well correlated, except in module 1 , in which $r=0.77$. The main significant difference was in the mean TA component of the SV, which was significantly different $(p=0.001)$. The correlations of the third and fourth NC between these two groups were low ( $r=0.49$ and $r=0.69$ respectively). The main differences were during the beginning of the stance phase (0-20\% of the cycle) and at the transition to the swing phase (50-65\% of the cycle). There was also a difference in the fifth NC ( $r=0.72)$. The activation of the ED was lower in the Contralateral leg than in the Ipsilateral leg at the transition between the stance and the swing phase ( $60 \%$ of the gait cycle). 


\section{Discussion}

This study deals with the investigation of the differences in the muscle activation patterns between healthy subjects (Control group) and subjects with ACL deficiency (Ipsilateral and Contralateral groups). The differences were studied at two levels: individual muscle timing patterns and muscle synergies. The novel contribution is the muscle synergy analysis in subjects with ACL deficiency, which we think it can provide valuable information during the rehabilitation treatment.

The results at the level of onset-offset patterns showed similarities with previous studies. Courtney et al. (2005) reported the onset and offset patterns of the Tibialis Anterior, Gastrocnemius Medialis, Medial Hamstrings and Quadriceps during fast-inclined walking in a treadmill for a Control group and an Adapter ACL-deficient group. Authors found that Tibialis Anterior and Medial Hamstrings had a longer activation period, which is in agreement with the presented results for the TA and ST muscles. They did not observe differences in Quadriceps (represented by RF in our study). Gastrocnemius Medialis pattern of the Adapter group (activated just before and after the heel strike) was different compared to our results obtained for the Ipsilateral and Contralateral GL (mainly activated during the mid stance phase). Nevertheless, this difference could be attributed to the fact that the gait was faster than in our study and there was an inclination.

Knoll et al. (2004) studied the gait adaptations at the level of muscle activations of subjects with ACL deficiency before and after the surgery. They measured EMG signals in Vastus Lateralis and Medialis, Adductor Longus and Biceps Femoris during walking. The activation-deactivation patterns obtained in their study are comparable to the ones in Figure 1. Overall, Ipsilateral's Vastus Lateralis and Biceps Femoris had a longer activation. In the work presented here, the results of the Biceps Femoris activation pattern are comparable to the ST pattern, which also belongs to Hamstrings. These results show longer co-contraction between Hamstrings and Quadriceps to stabilize the knee joint. 
2 flexor) and Quadriceps (knee extensor), are stabilizing the knee when the ACL is ruptured

3 (Chmielewski et al., 2001; Rudolph et al., 2001). These muscles control the joint to avoid

high displacements of the tibia with respect to the femur. There were also observable differences in muscles which control the ankle (SO, TA, GL and ED). The activation of these muscles was longer for the subjects with ACL deficiency. As Chmielewski et al. (2001) mentioned, there is a shift of support moment from the knee to the ankle which may suggest that there is a transfer of the leg control away from the injury.

At the level of muscle synergies, there were no statistical differences regarding the dimensionality of the signal factorization. In this case there were no observable differences in the number of modules needed to reconstruct the signals among groups, which represents that the control of the CNS is not more complex in subjects with ACL deficiency than in healthy subjects, in contrast with other studies which analyzed post-stroke subjects (Clark et al., 2010). The VAF values that evaluated the reconstruction of the EMG signal were similar among groups, either using 4 or 5 modules. However, this fact did not exclude that some differences could be detected between groups. Figure 2 showed that in all three groups, the VAF values of all muscles were higher than 0.9 using 5 modules, so the muscle synergy analysis was carried out using 5 synergies (Clark et al., 2010). The analysis of the differences intra-groups showed that all modules were overall independent from each other.

EMG data were collected at both legs simultaneously. Measuring left and right legs at the same time avoided the differences that could appear when measuring the EMG in two different cycles. EMG was normalized by MVC values, which allowed comparing the differences in the magnitude of the NCs (time-dependent synergy components). In Figures Figures 4 to 6 , it can be observed that NCs 1, 3, 4 and 5 were overall comprised between 0 and 0.4 and NC 2 was slightly higher (comprised between 0 and 0.62). 

that analyzed the muscle synergy components in healthy subjects. Oliveira et al. (2014) carried out a study to compare the influence of EMG processing (averaging, concatenation and the used number of cycles) in the muscle synergy factorization. They reported the results

for 5 modules. Our first 4 modules can be identified in 4 of their modules. The fifth module is different due to the fact that they did not measure ED signal (contained in module 5 in our case). Clark et al. (2010) carried out a study of the muscle synergy analysis with paretic and healthy subjects and Neptune et al. (2009) simulated gait using muscle activation modules. Both studies reported results for 4 modules that could be identified with our modules 1 to 4 . In all mentioned studies, EMG was normalized over all trials. In our study, EMG data were normalized by MVC values to evaluate differences in the magnitude of NCs.

Although the differences were small, SVs tended to be more similar between Control and Contralateral groups, suggesting that in those groups the CNS activates the same groups of muscles synergistically. However, it is not clear which groups presented comparable NCs. Modules 1 and 2 presented similar NCs between Contralateral and Control groups and modules 3 to 5 between Control and Ipsilateral groups. Therefore, the control of both legs of the injured subjects suffered small alterations compared to healthy subjects. Figures 4 to 6 show that the variability (represented by the standard deviation) in the NCs among subjects was higher for the injured subjects (Contralateral and Ipsilateral group). However, some different tendencies among groups can be observed. The second NC (which activated mainly SO and GL) in the Ipsilateral group presented two peaks during the stance phase and the NCs of modules 1 and 3 were higher at the beginning of early stance compared with the Control group. This could be caused by the fact that the subject tried to stabilize the joints of the Ipsilateral leg. Along similar lines of the observed results in the onset-offset patterns, Quadriceps and Hamstrings presented more co-contraction in the Ipsilateral group than in the 
1 Control one. In Figure 4, it can be observed that the fourth SV of the Ipsilateral group

2 presented weights higher for the RF and VL (Quadriceps) and lower for the ST (Hamstrings) than in the Control group. This fact yielded similar Quadriceps and Hamstrings weights in the Ipsilateral group, indicating higher co-contraction at the knee. Figure 6 shows that the peak of the module 5 (mainly related to ED activation) at the transition between the stance to swing phase was higher for the Ipsilateral and Control group than for the Contralateral group. The explanation of this result in module 5 could be two-fold. On the one hand, the control of the injured leg is transferred to the ankle, as mentioned in (Chmielewski et al., 2001), which is suggested with the comparison of Ipsilateral and Contralateral groups (also observed in onsetoffset patterns). On the other hand, the contralateral leg avoids providing a high acceleration to the body at toe off which would destabilize the injured leg during its initial stance, suggested by the smoother curve of the fifth $\mathrm{NC}$ of the contralateral leg compared to the one of the Ipsilateral leg.

Three main limitations of our study should be recognized. The first one is that, although the identification of the differences from a Control group could be useful to observe objective improvements during a rehabilitation treatment (to reinforce the clinical evaluation), a post-surgery follow-up study should be carried out to see how the muscle synergy components change along time. Some studies in the literature mention that the EMG pattern restores to levels similar to prior the injury (Ferber and Osternig, 2002; Knoll et al., 2004), but authors of these studies analyzed individual EMG signals and no synergy components. The second limitation is the sample size. With a wider sample, a study could be carried out to evaluate whether the subjects with ACL deficiency suffer other injuries or not. Comparing the muscle synergies with the observed tendencies from an ACL-deficient population could be useful to detect anomalies. The third limitation is related to the use of only one gait cycle. Due to space limitations, we only analyzed one gait trial. As mentioned, some separated gait 
1 trials were performed over the force plates and we picked the one with the cleaner data.

2 Processing more than one trial could reduce intra-groups variability yielding to more 3 conclusive results.

4

The main contribution of this study was the detection of slight differences in muscle synergy patterns of subjects with ACL deficiency during walking as compared to healthy subjects. In particular, the variations in NCs would explain the adaptations in the synergistic muscle patterns after an ACL rupture. In conclusion, our initial hypothesis where we suggested that differences in muscle synergy components may be observed in ACL-deficient and non-injured subjects was satisfied. Despite the similarities among groups, different trends were identified. The analysis of these muscle synergy tendencies can be useful as a follow-up study during a rehabilitation treatment. The follow-up of the adaptations of the synergy pattern might help the physiotherapist to know in more detail the progress of the rehabilitation.

Another important field of application is in motion simulation. Recently, some studies used muscle synergy components to decrease the indeterminacy when calculating the muscle forces in dynamic simulations of healthy subjects (Allen and Neptune, 2012; Neptune et al., 2009; Walter et al., 2014). Using muscle synergy components extracted from subjects with ACL deficiency could be also useful to predict muscle forces in gait dynamic analyses of those subjects. 


\section{References}

Allen JL, Neptune RR. 2012. Three-dimensional modular control of human walking. J Biomech. 45:2157-2163.

Button K, Van Deursen R, Price P. 2006. Classification of functional recovery of anterior cruciate ligament copers, non-copers, and adapters. British J Sport Med. 40:853-858.

Chmielewski TL, Rudolph KS, Fitzgerald GK, Axe MJ, Snyder-Mackler L. 2001. Biomechanical evidence supporting a differential response to acute ACL injury. Clin Biomech. 16:586-591.

Clark DJ, Ting LH, Zajac FE, Neptune RR, Kautz SA. 2010. Merging of healthy motor modules predicts reduced locomotor performance and muscle coordination complexity post-stroke. J Neurophisiol. 103:844-857.

Courtney C, Rine RM, Kroll PG. 2005. Central somatosensory changes and altered muscle synergies in subjects with anterior cruciate ligament deficiency. Gait Posture. 22:69-74.

De Groote F, Jonkers I, Duysens J. 2014. Task constraints and minimization of muscle effort result in a small number of muscle synergies during gait. Front Comput Neurosci. 8:1-11.

De Rugy A, Loeb GE, Carroll TJ. 2013. Are muscle synergies useful for neural control?. F Comput Neurosci. 7:19.

Dominici N, Ivanenko YP, Cappellini G, D’Avella A, Mondì V, Cicchese M, Fabiano A, Silei T, Di Paolo A, Giannini C, Poppele RE, Lacquaniti F. 2011. Locomotor primitives in newborn babies and their development. Science. 334:997-999.

Erdemir A, McLean S, Herzog W, Van den Bogert AJ. 2007. Model-based estimation of muscle forces exerted during movements. Clin Biomech. 22:131-154.

Ferber R, Osternig LR, Woollacott MH, Wasielewski NJ, Lee JH. 2002. Gait mechanics in chronic ACL deficiency and subsequent repair. Clin Biomech. 17:274-285. 
1 Houck JR, Wilding GE, Gupta R, De Haven KE, Maloney M. 2007. Analysis of EMG patterns of control subjects and subjects with ACL deficiency during an unanticipated walking cut task. Gait Posture. 25:628-638.

Ivanenko YP, Cappellini G, Dominici N, Poppele RE, Lacquaniti F. 2005. Coordination of locomotion with voluntary movements in humans. J Neurosci. 25:7238-7253.

Ivanenko YP, Poppele RE, Lacquaniti F. 2004. Five basic muscle activation patterns account for muscle activity during human locomotion. J Neurophysiol. 556:267-282.

Kendall FP, McCreary EK, Provance PG, Rodgers MM, Romani WA. 2005. Muscles, testing and function: with posture and pain. 5th ed. Philadelphia: Lippincott Williams \& Wilkins.

Knoll Z, Kiss RM, Kocsis L. 2004. Gait adaptation in ACL deficient patients before and after anterior cruciate ligament reconstruction surgery. J Electromyogr Kines. 14:287-294.

Lacquaniti F, Ivanenko YP, Zago M. 2012. Patterned control of human locomotion. J Physiol. 590:2189-2199.

Lee DD, Seung HS. 2000. Learning the parts of objects by non-negative matrix factorization. Nature. 401:788-791.

Neptune RR, Clark DJ, Kautz SA. 2009. Modular control of human walking: a simulation study. J Biomech. 42:1282-1287.

Oliveira AS, Gizzi L, Farina D, Kersting UG. 2014. Motor modules of human locomotion: influence of EMG averaging, concatenation, and number of step cycles. Front Hum Neurosci. 8:335.

Renström PA. 2013. Eight clinical conundrums relating to anterior cruciate ligament (ACL) injury in sport: recent evidence and a personal reflection. Brit J Sport Med. 47:367-372. 
1 Rudolph KS, Axe MJ, Buchanan TS, Scholz JP, Snyder-Mackler L. 2001. Dynamic stability in the anterior cruciate ligament deficient knee. Knee Surg, Sport Tr A. 9:62-71.

3 Serrancolí G, Font-Llagunes JM, Barjau A. 2014. A weighted cost function to deal with the muscle force sharing problem in injured subjects: A single case study. P I Mech Eng K J Mul. 228:241-251.

Ting LH. 2007. Dimensional reduction in sensorimotor systems: a framework for understanding muscle coordination of posture. Prog Brain Res. 165:299-321.

Ting LH, Chvatal SA, Safavynia SA, Mckay JL. 2012. Review and perspective: neuromechanical considerations for predicting muscle activation patterns for movement. Int J Numer Method Biomed Eng. 28:1003-1014.

Ting LH, Macpherson JM. 2005. A limited set of muscle synergies for force control during a postural task. J Neurophysiol. 93:609-613.

Ting LH, McKay JL. 2007. Neuromechanics of muscle synergies for posture and movement. Curr Opin Neurobiol. 17:622-628.

Walter JP, Kinney AL, Banks SA, D’Lima DD, Besier TF, Lloyd DG, Fregly BJ. 2014. Muscle synergies may improve optimization prediction of knee contact forces during walking. J Biomech Eng T ASME. 136:021031. 
1 Table 1. Cross correlation of the mean NC curves and SV values among modules for all three 2 groups.

3 Control

\begin{tabular}{|l|l|l|l|l|}
\hline \multicolumn{5}{|c|}{ NC } \\
\hline & 1 & 2 & 3 & 4 \\
\hline 2 & 0.20 & & & \\
\hline 3 & 0.16 & -0.48 & & \\
\hline 4 & $\mathbf{0 . 5 2}$ & -0.21 & $\mathbf{0 . 5 8}$ & \\
\hline 5 & -0.58 & -0.06 & -0.19 & -0.34 \\
\hline
\end{tabular}

\begin{tabular}{|l|l|l|l|l|}
\hline \multicolumn{5}{|c|}{ SV } \\
\hline & 1 & 2 & 3 & 4 \\
\hline 2 & 0.05 & & & \\
\hline 3 & 0.18 & -0.23 & & \\
\hline 4 & 0.06 & -0.36 & -0.05 & \\
\hline 5 & -0.38 & -0.13 & -0.07 & -0.38 \\
\hline
\end{tabular}

$4 \quad$ Ispilateral

\begin{tabular}{|l|l|l|l|l|}
\hline \multicolumn{5}{|c|}{ NC } \\
\hline & 1 & 2 & 3 & 4 \\
\hline 2 & 0.05 & & & \\
\hline 3 & $\mathbf{0 . 3 4}$ & -0.59 & & \\
\hline 4 & $\mathbf{0 . 5 6}$ & -0.26 & 0.30 & \\
\hline 5 & -0.5 & -0.31 & 0.29 & -0.14 \\
\hline
\end{tabular}

\begin{tabular}{|l|l|l|l|l|}
\hline \multicolumn{5}{|c|}{ SV } \\
\hline & 1 & 2 & 3 & 4 \\
\hline 2 & -0.26 & & & \\
\hline 3 & -0.23 & -0.10 & & \\
\hline 4 & 0.03 & -0.59 & 0.14 & \\
\hline 5 & -0.26 & -0.31 & -0.17 & -0.41 \\
\hline
\end{tabular}

5 Contralateral

\begin{tabular}{|l|l|l|l|l|}
\hline \multicolumn{5}{|c|}{$\mathrm{NC}$} \\
\hline & 1 & 2 & 3 & 4 \\
\hline 2 & 0.04 & & & \\
\hline 3 & $\mathbf{0 . 3 7}$ & -0.33 & & \\
\hline 4 & $\mathbf{0 . 8 2}$ & -0.29 & 0.18 & \\
\hline 5 & -0.43 & -0.12 & -0.11 & -0.26 \\
\hline
\end{tabular}

\begin{tabular}{|l|l|l|l|l|}
\hline \multicolumn{5}{|c|}{ SV } \\
\hline & 1 & 2 & 3 & 4 \\
\hline 2 & -0.16 & & & \\
\hline 3 & 0.20 & -0.14 & & \\
\hline 4 & -0.09 & -0.40 & -0.26 & \\
\hline 5 & -0.09 & -0.09 & 0.22 & -0.58 \\
\hline
\end{tabular}

$6 \quad$ Bold values indicate that there is significant positive correlation ( $p$-value $<0.001$ ) 
1 Table 2. Correlation of the mean NC curves and SV values among modules across groups.

\begin{tabular}{|c|c|c|c|c|c|}
\hline \multicolumn{7}{|c|}{$\mathrm{NC}$} \\
\hline Ipsilat. & 1 & 2 & 3 & 4 & 5 \\
\hline 1 & $\mathbf{0 . 7 7}$ & & & & \\
\hline 2 & -0.04 & $\mathbf{0 . 9 4}$ & & & \\
\hline 3 & 0.19 & -0.63 & $\mathbf{0 . 6 9}$ & & \\
\hline 4 & $\mathbf{0 . 6 9}$ & -0.28 & 0.30 & $\mathbf{0 . 8 6}$ & \\
\hline 5 & -0.42 & 0.01 & 0.11 & -0.07 & $\mathbf{0 . 8 1}$ \\
\hline \multicolumn{7}{|c|}{} \\
\hline
\end{tabular}

\begin{tabular}{|c|c|c|c|c|c|}
\hline \multicolumn{7}{|c|}{ SV } \\
\hline $\begin{array}{c}\text { Control } \\
\text { Ipsilat. }\end{array}$ & 1 & 2 & 3 & 4 & 5 \\
\hline 1 & 0.80 & & & & \\
\hline 2 & -0.36 & $\mathbf{0 . 9 9}$ & & & \\
\hline 3 & -0.18 & -0.28 & $\mathbf{0 . 9 6}$ & & \\
\hline 4 & 0.28 & -0.35 & -0.17 & 0.70 & \\
\hline 5 & -0.32 & -0.20 & 0.03 & -0.47 & $\mathbf{0 . 9 6}$ \\
\hline
\end{tabular}

\begin{tabular}{|c|c|c|c|c|c|}
\hline \multicolumn{7}{|c|}{$\mathrm{NC}$} \\
\hline $\begin{array}{c}\text { Control } \\
\text { Contralat. }\end{array}$ & 1 & 2 & 3 & 4 & 5 \\
\hline 1 & $\mathbf{0 . 7 9}$ & & & & \\
\hline 2 & 0.01 & $\mathbf{0 . 9 2}$ & & & \\
\hline 3 & 0.24 & -0.62 & $\mathbf{0 . 4 3}$ & & \\
\hline 4 & $\mathbf{0 . 7 0}$ & -0.20 & 0.08 & $\mathbf{0 . 8 4}$ & \\
\hline 5 & -0.44 & 0.12 & -0.36 & -0.30 & $\mathbf{0 . 7 3}$ \\
\hline
\end{tabular}

\begin{tabular}{|c|c|c|c|c|c|}
\hline \multicolumn{7}{|c|}{ SV } \\
\hline $\begin{array}{c}\text { Control } \\
\text { Contralat. }\end{array}$ & 1 & 2 & 3 & 4 & 5 \\
\hline 1 & $\mathbf{0 . 9 3}$ & & & & \\
\hline 2 & -0.22 & $\mathbf{0 . 9 9}$ & & & \\
\hline 3 & 0.38 & -0.31 & $\mathbf{0 . 9 5}$ & & \\
\hline 4 & 0.10 & -0.31 & -0.17 & 0.91 & \\
\hline 5 & -0.28 & -0.17 & 0.10 & -0.43 & $\mathbf{0 . 9 6}$ \\
\hline
\end{tabular}

3

\begin{tabular}{|c|c|c|c|c|c|}
\hline \multicolumn{7}{|c|}{$\mathrm{NC}$} \\
\hline Ipsilat & 1 & 2 & 3 & 4 & 5 \\
\hline 1 & $\mathbf{0 . 9 8}$ & & & & \\
\hline 2 & 0.08 & $\mathbf{0 . 9 6}$ & & & \\
\hline 3 & $\mathbf{0 . 3 6}$ & -0.60 & $\mathbf{0 . 4 9}$ & & \\
\hline 4 & $\mathbf{0 . 5 9}$ & -0.19 & 0.10 & $\mathbf{0 . 6 9}$ & \\
\hline 5 & -0.50 & -0.25 & -0.26 & -0.31 & $\mathbf{0 . 7 2}$ \\
\hline
\end{tabular}

\begin{tabular}{|c|c|c|c|c|c|}
\hline \multicolumn{7}{|c|}{ SV } \\
\hline $\begin{array}{c}\text { Ipsilat. } \\
\text { Contralat. }\end{array}$ & 1 & 2 & 3 & 4 & 5 \\
\hline 1 & 0.77 & & & & \\
\hline 2 & -0.19 & $\mathbf{0 . 9 9}$ & & & \\
\hline 3 & 0.36 & -0.13 & $\mathbf{0 . 9 6}$ & & \\
\hline 4 & 0.07 & -0.60 & 0.23 & 0.82 & \\
\hline 5 & -0.38 & -0.28 & -0.10 & -0.32 & 0.87 \\
\hline
\end{tabular}

$4 \quad$ Bold values indicate that there is significant positive correlation ( $p$-value $<0.001)$ 


\section{Figure captions}

2 Figure 1. Muscle activation-deactivation patterns during the gait cycle for the three groups:

3 Control, Contralateral, Ipsilateral. The gait cycle is comprised between one heel strike $(0 \%)$

4 and the following one of the same leg (100\%).

5 Figure 2. Mean VAF values of all muscles using 4 (left) and 5 modules (right). Black vertical

6 lines represent standard deviations from the mean values. Horizontal dashed black line stands

7 for the threshold of $\mathrm{VAF}=0.9$.

8 Figure 3. Synergy components decomposing EMG signals in 5 modules. NCs for all subjects

9 (in grey, left) with their mean values (thick black lines) and standard deviations (dashed

10 lines). SVs for all subjects in descend order (in grey, right) with their mean values (in black

11 bars) and standard deviations (in error black bars). The gait cycle is comprised between one heel strike (0\%) and the following one of the same leg (100\%).

Figure 4. NCs (left) and SVs (right) for Control and Ipsilateral groups. The thick lines in the left plots represent the mean value of the NCs and dashed lines one standard deviation from the mean. In the right plots, the bars represent the mean value of the SVs for all subjects and the black error bars their standard deviations. The gait cycle is comprised between one heel strike $(0 \%)$ and the following one of the same leg (100\%).

Figure 5. NCs and SVs for Control and Contralateral groups. Refer to Figure 4 for meaning of thick and dashed lines as well as the error bars.

Figure 6. NCs and SVs for Ipsilateral and Contralateral groups. Refer to Figure 4 for meaning of thick and dashed lines as well as the error bars. 


\section{Potential Reviewers}

\section{$\underline{\text { Miguel Tavares da Silva }}$}

Professor at IDMEC-Instituto de Engenharia Mecânica, Instituto Superior Técnico, Universidade de Lisboa, Lisbon, Portugal.

Email: miguelsilva@tecnico.ulisboa.pt

\section{$\underline{\text { Friedl De Groote }}$}

Postdoctoral research scientist at the Department of Mechanical Engineering in KU Leuven, Leuven, Belgium.

friedl.degroote@kuleuven.be

\section{$\underline{\text { Maxime Raison }}$}

Assistant Professor at the Department of Mechanical Engineering in Polythecnique Montréal, Montreal, Canada.

maxime.raison@polymtl.ca

\section{$\underline{\text { Massimo Sartori }}$}

Postdoctoral research scientist at the Department of Neurorehabilitation Engineering, University Medical Center Goettingen, Goettingen, Germany.

massimo.srt@gmail.com 


\section{Figure 1}

TA

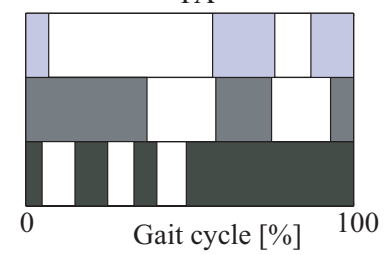

RF

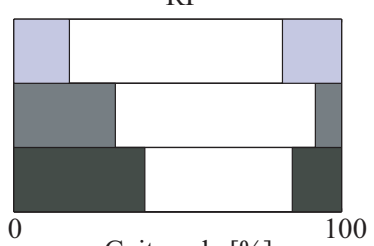

Gait cycle [\%]
SO

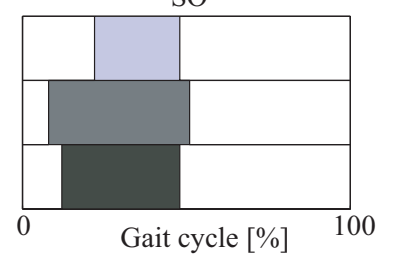

VL

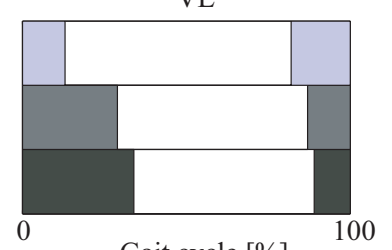

GL

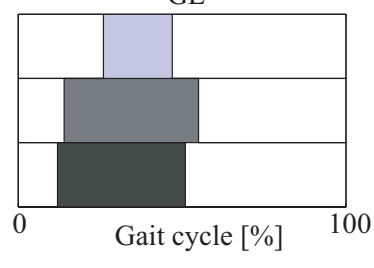

ST

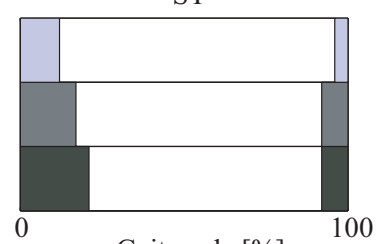

Gait cycle [\%]

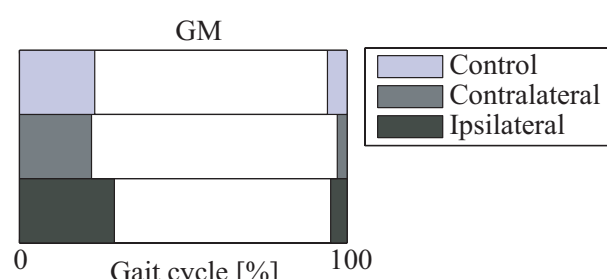

ait cycle [\%]

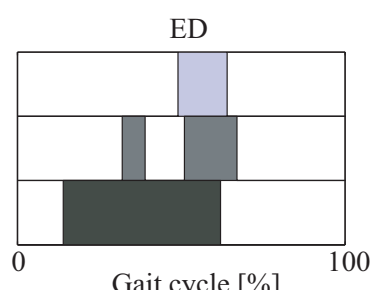




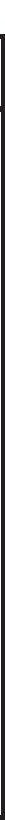

Figure 2 
Figure 4
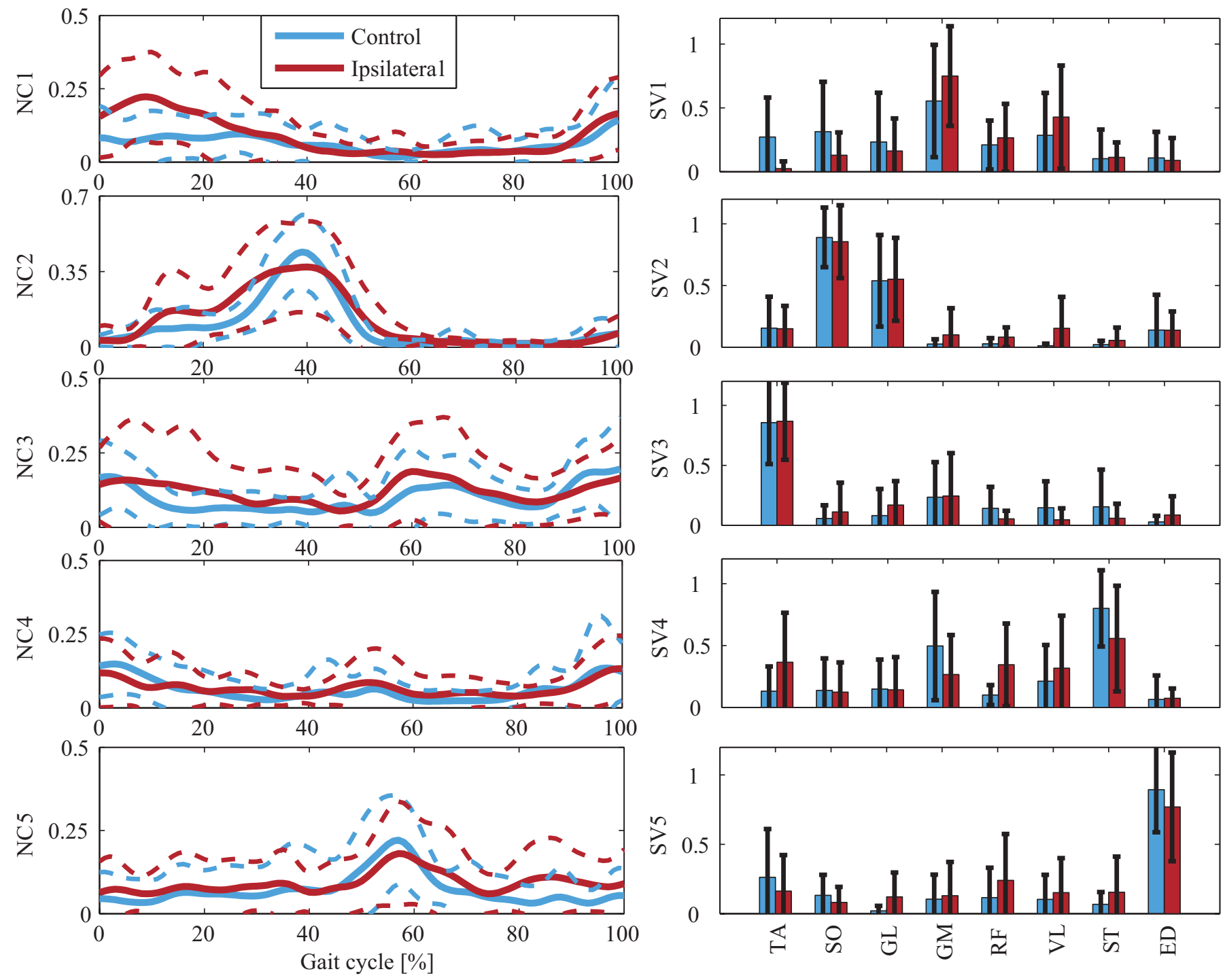
Figure 5
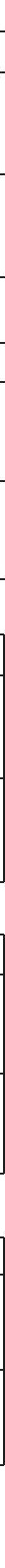

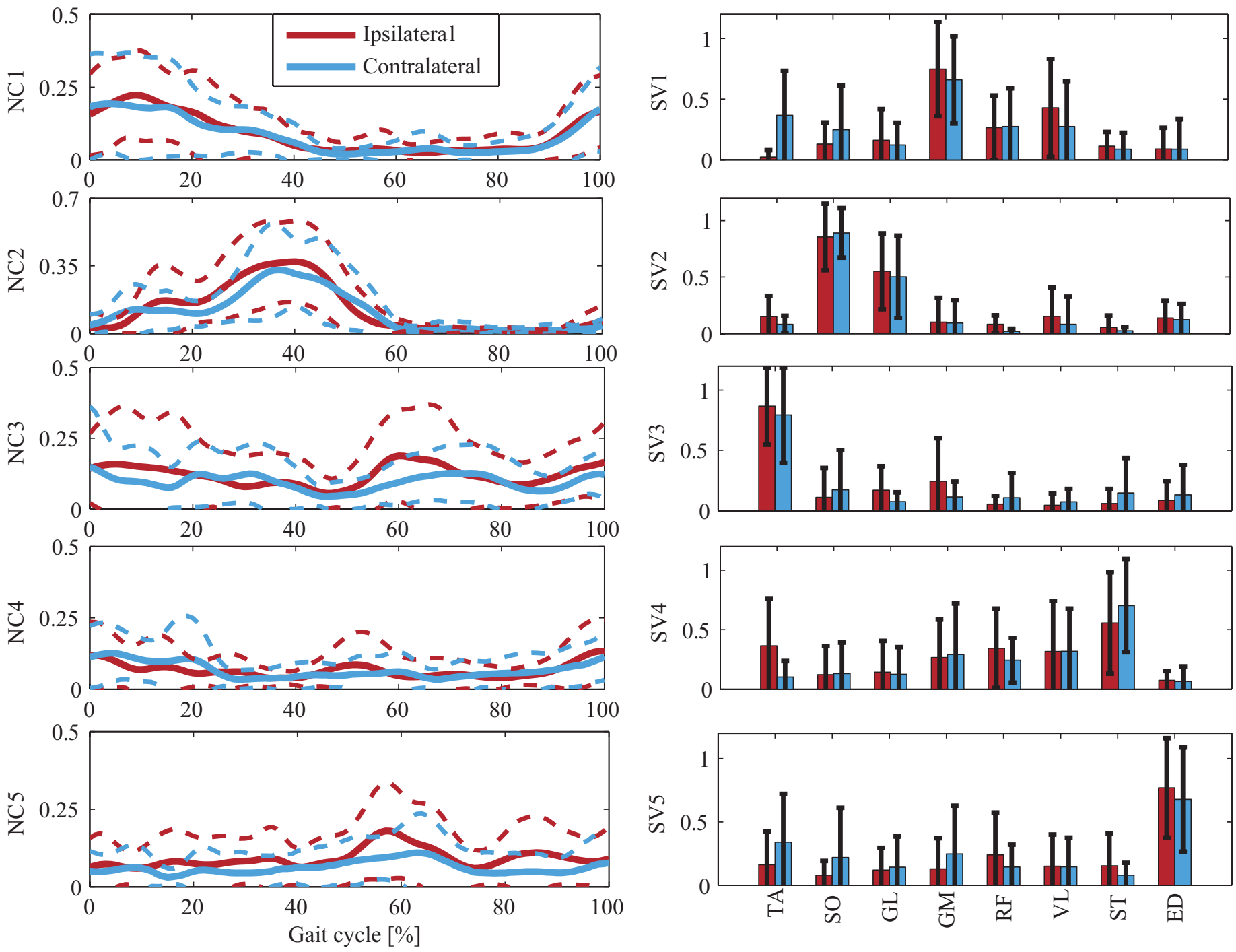
Supplementary Material
Click here to download Supplementary Material: Supplementary_data.xls

\begin{abstract}
Click here to download Supplementary Material: Supplementary_data.xls
\end{abstract}

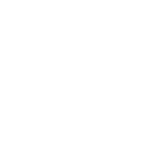

